\title{
INDICATONS FOR ADENOMASTECTOMY IN THE PAST 10 YEARS: WHAT HAS CHANGED THE DECISION OF THE SURGEON?
}

\author{
Marina Sonagli', Eduardo Bertolli', Hirofumi lyeyasy', Fabiana Baroni Alves Makdissi \\ ${ }^{1}$ Ac Camargo Cancer Center - São Paulo (SP), Brazil.
}

Introduction: Adenomastectomy (AM) consists of the almost complete removal of the mammary gland, but preserving the skin envelope and areolar-papillary complex (APC). This technique was initially indicated to women with family history of breast cancer and for treating fibrocystic breast disease. Currently, it can be carried out in a prophylactic manner in patients with genetic mutations associated with higher risks of breast cancer, and in a therapeutic manner for the treatment of tumors smaller than $3 \mathrm{~cm}$, which are peripheral or more than $2 \mathrm{~cm}$ away from the APC, with negative retroareolar margin intraoperative freezing. Among the advantages of keeping the APC in AM are the cosmetic results, which favor self-image and self-esteem. Objectives: to assess the incidence of prophylactic AM (PAM) and therapeutic AM (TAM) and relate the PAM with the presence of family history of neoplasms, genetic changes that present high risk for breast cancer or desire of the patient (absence of these criteria). Methods: retrospective study of patients submitted to AM at A.C. Camargo between 2007 and 2017. Results: two-hundred and ninety patients were included, of which 77 were submitted to bilateral AM (154 AM), and 213 patients, to unilateral AM, accounting for 367 AM. Between 2007 and 2009, 116 PAM and 20 TAM were performed; between 2010 and 2013, 40 PAM and 32 TAM; and between 2014 and 2017, 71 PAM and 88 TAM. The indications of PAM were: between 2007 and 2009, 7 PAM were owed to pathogenic mutations, 50 were due to "family history of high risk", and 58 due to the patient's desire; between 2010 and 2013, 14 were caused by pathogenic mutations, 12 by "family history of high risk", and 14 by the patient's desire; between 2014 and 2017, 45 were owed to pathogenic mutations, 18 to "family history of high risk", and 8 to the desire of the patient. Conclusions: it is observed that between 2007 and 2017 there was a progressive increase in the incidence of TAM, especially after 2010; simultaneously, there was a reduction in PAM, and they were finally equivalent in 2017. It is possible to observe that the reduction in the incidence of PAM in the first triennium was owed to the reduction in indications of PAM, based only on family history or on the patient's desire. The recapture of PAM occurred with the increasing number of diagnosis of hereditary syndromes. At the end of the decade, most of the PAMs were performed due to the presence of pathogenic mutations, as advised by NCCN: 